\title{
Interventional radiology and COVID-19: evidence-based measures to limit transmission
}

\author{
Poornima Elizabeth Chandy \\ Muhammad Umer Nasir (1) \\ Sivasubramanian Srinivasan (1) \\ Darren Klass (1) \\ Savvas Nicolaou (i) \\ Suresh B. Babu (1)
}

From the Departments of Emergency and Trauma Radiology (P.E.C. $\bowtie$ liztom78@gmail.com, M.U.N., S.N.) and Interventional Radiology (D.K.) Vancouver General Hospital, University of British Columbia, Canada; Department of Diagnostic Radiology (S.S. S.B.B.) NHG, Khoo Teck Puat Hospital, 90 Yishun Central, Singapore.

Received 22 March 2020; accepted 24 March 2020.

Published online 27 March 2020.

DOI 10.5152/dir.2020.20166

\section{ABSTRACT}

As we face an explosion of COVID-19 cases and deal with an unprecedented set of circumstances all over the world, healthcare personnel are at the forefront, dealing with this emerging scenario. Certain subspecialties like interventional radiology entails a greater risk of acquiring and transmitting infection due to the close patient contact and invasive patient care the service provides. This makes it imperative to develop and set guidelines in place to limit transmission and utilize resources in an optimal fashion. A multi-tiered approach needs to be devised and monitored at the administrative level, taking into account the various staff and patient contact points. Based on these factors, work site and health force rearrangements need to be in place, while enforcing segregation and disinfection parameters. We are putting forth an all-encompassing review of infection control measures that cover the dynamics of patient care and staff protocols that such a situation demands of an interventional department.

$\mathrm{T}$ he ongoing COVID-19 outbreak caused by a novel Corona virus known as SARSCoV-2, has become a global pandemic with more than 270000 cases reported worldwide at the time of this article, with number of deaths more than 11000 . With the pathogen being a novel virus, many aspects of the organism and manifestations related to acute and long-term consequences are still unknown. The virus characteristics, mutagenic forms, origin and routes of animal to human transmission, mode of human spread, extent of asymptomatic carriers, variables affecting mortality, effective treatment options and feasibility of developing vaccine are all parameters which need further study and definition. As other departments, it is imperative on Interventional Radiology (IR) to provide its services safely and effectively while reducing the risk of transmission to the staff. The virus has been shown to have phylogenetic similarity as well as severity of manifestations comparable to severe respiratory syndrome (SARS) caused by SARS-CoV-1. With much more yet to be known about the virus, an adequate protocol needs to be derived from the available fragmentary data and lessons learnt from prior outbreaks like SARS. We aim to put forth guidelines that the service needs to adopt to maintain a balance between optimal patient care without compromising on precautionary measures for IR staff.

\section{IR departmental policies and actions}

Most secondary transmission of SARS and MERS occurred in the hospital setting. This has been true for COVID-19 as well. In a report of 44672 COVID-19 cases in China, 1716 were health workers (3.8\%), and $14.8 \%$ of confirmed cases among health workers were classified as severe or critical and 5 deaths were observed (1).

Protective measures should be in place before and upon the patient's arrival in the department, throughout the hospital stay, and during room disinfection following the patient's discharge. It is particularly important to protect individuals at increased risk of infection including other patients and healthcare personnel (HCP).

\section{Vetting and prioritization}

The risk of transmission is directly related to the degree of contact between the public and HCP. Therefore, interventional radiologists are predisposed to a substantial risk of ac- 
quiring infection. The first step in limiting the propagation of the disease is proper vetting of the requested interventional procedures and postponement of the elective procedures till the current situation is brought under control. The interventional radiologist should limit acceptance of cases to urgent and semi urgent ones. More liberal adoption of noninvasive practices may be initiated to manage inpatients awaiting interventions as well as those presenting to the emergency department (2).

\section{Elective procedures}

All patients who have been scheduled for elective procedures should be contacted by the nursing staff and instructed to call ahead if they have any symptoms suggestive of COVID-19 or have travelled to the endemic areas. Depending on the situation, the decision may be made to defer all elective procedures till further notice.

\section{Urgent procedures}

For patients requiring urgent intervention, the requesting clinical team must be instructed to assess the patients for fever, myalgia, or respiratory symptoms and to follow preventive measures like making the patient wear a facemask and to sanitize the contact surfaces as much as reasonably possible. Emergency medical services should inform the receiving healthcare facility of suspected or confirmed COVID-19 cases and follow local or regional transport protocol. Effective communication in a timely fashion may allow sufficient time for the facility to prepare for the receipt of such patients (3).

Control of patient and staff movement

- Limiting all traffic through the healthcare facility is paramount in establishing control.

- The entry point of all confirmed cases should be limited to one designated section.

- Signs and posters are recommended at these points detailing instructions on hand hygiene and cough etiquette, appropriate use and disposal of masks.

- Adequate alcohol-based hand sanitizers and receptacles for waste disposal should be placed in these areas.

- Where appropriate, physical barriers should be installed to limit interpersonal contact.

- Rapid assessment and isolation of highrisk patients in an examination room is advocated.
- No more than one visitor should be allowed to accompany the patient after the visitor has been assessed regarding travel history and clinical symptoms. If found to have affirmative history, the visitor should be asked to leave the premises.

These recommendations include limiting not only patients and staff, but also accessory regions functioning within the hospital including canteens, shops, security, support offices and help desks. All subsections not necessary for critical functioning of the unit or hospital should be temporarily suspended. In those areas which are deemed necessary, the minimal workforce to keep the service afloat is recommended.

\section{Workflow enhancements}

With restrictions in place, greater team play and coordinated effort is needed for smooth and effective functioning of the department. A well-planned and rehearsed approach should be put in place by a core group in charge of the unit. Each member of the interventional team should have a clear understanding of the role they play so that procedures can be undertaken with the least amount of stress and confusion while limiting unnecessary cross interactions.

\section{Reinforcement of departmental protocols including infection control}

The interventional team should designate members who will be deemed responsible for communication with the hospital management and public health officials for development and implementation of infection control measures and to disseminate information to the team.

The core response team should be responsible for coordinating with the Regional and Institutional healthcare policies while deciding on a protocol suited specifically to match the needs of IR. Since IR, unlike most other subspecialties of Radiology, deals closely with patients and support staff, infection control steps should be strictly initiated and followed. All appropriate equipment necessary for interacting with patients confirmed to have COVID-19 should be provided to involved staff while ensuring that personal hygiene practices are enforced. Situational awareness and constant updating of the team on changing policies ensures faith in the response team and prevents untoward exposure.
Stay at home policy for IR staff or staff returning from infected areas

Lessons are to be learnt from Taiwan government's prompt and proactive 124 step protocol. These steps included integration of individual travel history with government identification cards so that all individuals with travel abroad could be recognized and assessed. All travelers were made to complete a health declaration form which was linked to an online form, either prior to departure from or upon arrival at a Taiwan airport. After assessment, those identified as high risk were put under mandatory home quarantine and monitored electronically to ensure quarantine was followed. The Taiwanese government also allowed all hospitals, clinics, and pharmacies in Taiwan the access to patients' travel histories (4).

These timely and stringent measures resulted in Taiwanese government being able to contain the outbreak to less than 50 , successfully dispelling predictions of Taiwan getting the second highest number of cases due to proximity and number of flights with China.

Regulations are already in place for most jurisdictions across the world. Any suspicion of contact with an infected patient or travel to a region with COVID invites a mandatory self-isolation for at least 2 weeks. There may however be lapses or refusal to comply with existing recommendations on the part of staff members. The details of the protocol in place should be clearly conveyed to all team members. Consideration may also be given to mobile monitoring to ensure that the isolation steps are complied with.

\section{Cleaning and disinfection procedures}

All work stations, IR suites, departmental areas in use should be cleaned and disinfected prior to and after a new team member uses it. Apart from this, mandatory cleaning should be undertaken 4 times a day to reduce any transmission risk.

All nondisposable medical equipment should be cleaned and disinfected according to manufacturer's instructions and facility policies. Environmental protection agency (EPA)-registered disinfectants that have qualified under EPA's emerging viral pathogens program for use against SARSCoV-2 should be used.

Routine cleaning and disinfection procedures (e.g., using cleaners and water to pre-clean surfaces prior to applying an EPA-registered, hospital-grade disinfectant to frequently touched surfaces for appropriate contact times as indicated on the 
product's label) are appropriate for SARSCoV-2 in healthcare settings.

Management of laundry, food service utensils, and medical waste should also be performed in accordance with routine procedures (3).

\section{Role of leadership and direction}

The person in charge of the IR section has to strike a balance between providing patient care while assessing the risk profile and keeping the safety of his team in mind. This will result in tough decisions having to be made which may find resistance from clinical teams and management. Opting out of planned elective procedures is liable to earn the criticism of the teams caring for the patient and the patients themselves. The allocated resources have to be prudently distributed and steps should be taken to ensure optimization of valued protective equipment. With many areas facing shortages, alternatives to N95s should be considered, including other filtering facepiece respirators, elastomeric half-mask and full facepiece air purifying respirators, and powered air purifying respirators (PAPRs). Extra care should be taken to ensure that respirators are reserved for aerosol-generating procedures. Transparent decision making, clear communication and stringent policy implementation is imperative in infection control and keeping HCP safe. The team leader also needs to be up to date with evolving scenarios while adjusting existing protocols and decisions to match constantly a changing ground reality.

\section{IR patient-related actions}

\section{Checking of patients}

Clinical samples should be collected from suspected cases and extraction of nucleic acids should be performed with a High Pure Viral Nucleic Acid Kit. These extracted nucleic acid samples should subsequently be tested for viruses and bacteria by polymerase chain reaction (PCR), in accordance with manufacturer instructions. Reverse transcription PCR (RT-PCR) assay should then be used to detect viral RNA by targeting a consensus RdRp region of pan $\beta-\operatorname{CoV}(5)$.

\section{Segregation of patients}

The clinical teams should be clearly directed to let the IR team know when a procedure is being requested for a confirmed COVID-19 patient. These patients should be separated into different groups based on risk of transmission. The procedures should then be performed in isolated locations so as to mitigate the transmission of highly infective groups to others awaiting treatment.

Depending on the burden of cases and resources of the institution, a dedicated COVID-19 care section with isolated floor space and designated HCP may be established.

Any patient with confirmed or suspected COVID-19 should be placed in a single-person room with a dedicated bathroom with the door closed at all times. Patients should be confined to the same room for the extent of their stay.

Suspected unconfirmed patients should not be housed in the same unit or room as a confirmed case.

The patients should be instructed to wear facemasks at all times to reduce source transmission. Those unable to tolerate facemasks should use tissues to cover the mouth and nose.

Patient movement outside designated units should be limited, and portable imaging and lab work should be performed in the room when feasible.

Following discharge or transfer, entry of all personnel into the room should be allowed only after sufficient time has elapsed for air changes to remove potentially infectious particles.

\section{IR in COVID-19 patients}

Basic precautions and vascular access

As instituted in guidelines by CDC and OSHA, SIR recommends the use of protective eyewear, facemask, gowns, and shoe covers in the event of droplet or splash risk. Maximum barrier precautions (cap, mask, sterile gown, sterile gloves, double glove use, and large sterile drape) is advised during the insertion of central venous catheters and all invasive interventional procedures. Two-handed needle recapping technique should be avoided and if re-capping is indicated, a re-capping device or a one-handed method is advised. The use of a designated sink for bodily fluid disposal and use of personal protective equipment (PPE) by IR staff during the disposal process is also advised (6).

It is recommended that all attempts at vascular access be made with ultrasonography (US) guidance to increase the chance of access at first attempt. Central catheter placement should be performed in an isola- tion suite with the use of a portable US and a C-arm. Single-use sterile ultrasound gel is recommended. Suturing should be avoided as it increases the risk of oozing at the catheter entry site. The US unit and transducer should have an extra-long cord and should be double-bagged. The $\mathrm{C}$-arm also requires two layers of waterproof plastic. The US images should ideally be transmitted wirelessly to the archive or a wired connection to the archive should be available in an antechamber outside of the patient's room (7).

\section{IR procedure room}

Level 1 procedures (guided, diagnostic and/or therapeutic punctures, aspiration and biopsy) are generally performed in an isolated radio-diagnostic room. The disposable protection must be changed and the examination table systematically disinfected between each patient. An aerosol technique must be used to disinfect potentially contaminated IR surfaces and equipment which are not easily accessible. All waste must be eliminated at the end of each procedure. Inadvertent entry to this room must be prevented. The room must be equipped with a point for washing and disinfecting hands and contain as few consumables as possible.

Level 2 and 3 procedures (e.g., angiography, embolization, stent insertion, ablation, drainage, inferior vena cava filter, central line placement, endoprosthesis, neurointerventions) need to be performed in a specially created room, analogous to an operating theatre. If such a room cannot be set aside for isolation and subset of COVID-19 patients, a separate structure should be created within a reasonable time. This area must be separate from the rest of the department with a controlled environment and access, compatible with an ISO 7 level of performance. Inside this, the different rooms must be organized depending on the differing levels of infection risk.

Post-intervention observation room must be close to the intervention room and rules of hygiene for this part of the area are the same as those for the intervention area (8).

\section{Infected patients' procedures in the department}

All procedures on confirmed COVID-19 cases should be undertaken in single-care isolation rooms. Ideally, airborne infection isolation rooms (AIIR), respirators and facemasks are core practices to be adopted 
when performing a procedure on a confirmed COVID-19 patient. AlIRs are especially recommended in aerosol generating procedures.

These are single-patient negative pressure rooms with a minimum of 6 air changes per hour. Air from these rooms should be exhausted to the outside or filtered through a high-efficiency particulate air (HEPA) filter before recirculation. Entry and exit to these rooms should be kept to a minimum and doors should be closed at all times except when needed for entry or exit. Monitoring and documenting of negative pressure to ensure adequacy should be put in place.

Only personnel essential to the procedure should be allowed in. All HCP in the room should wear an N95 or higher-level respirator, eye protection, gloves, and gown. Respirators for use in healthcare must be certified by the CDC/NIOSH. The HCP should be fit-tested if using respirators with tight-fitting facepieces (e.g., a NIOSH-approved N95 respirator) and trained in the proper use, safe removal and disposal, and medical contraindications (3).

The room should be promptly cleaned and disinfected after adequate time for air change has elapsed.

\section{Specimen collection}

Leak-proof plastic containers are recommended by WHO over glass specimen containers for specimen collection. There should not be any visible contamination on the external surface of the container. Containers should be carefully labelled to not only ensure that the correct specimen has been placed but also indicating the contagious nature of the specimen. The laboratory requisition should be placed in separate waterproof chamber. The Allegheny Health Network guidelines recommend that HCP wear gloves when handling the specimen container and specimen. Specimen containers should be transported in a Ziploc-type bag (S. C. Johnson \& Son) marked with a biohazard symbol. Body tissue specimens (i.e., biopsy specimens), body fluid specimens greater than $90 \mathrm{~mL}$, and cerebrospinal fluid specimens cannot be transported through a pneumatic tube transportation system (9).

\section{Equipment decontamination}

Decontamination of noncritical items such as the magnetic resonance imaging (MRI) or computed tomography (CT) gan- try, noninvasive ultrasound probes, blood pressure cuffs, and the viewing station keyboard and mouse should occur after every use (9). Critical items (endovascular or endovaginal ultrasound probes and reusable surgical instruments) require steam-sterilization of heat-resistant items after every use while heat-sensitive items can be sterilized with ethylene oxide gas, hydrogen peroxide gas plasma, ozone, or liquid chemical sterilant.

\section{IR staff-related actions}

\section{Reinforcement of basic hygiene}

In any contagion, HCP face a high degree of infection themselves. With high infectivity of COVID-19 and with asymptomatic carriers being transmitters, COVID-19 patients pose a high degree of infection to IR staff since they are involved directly in examining, interacting and treating patients. Being a respiratory infection with droplet and contact transmission, it is essential that handwashing and other infection prevention and control measures are in place. These HCP are at greater risk of infection and then become the inadvertent carriers to patients who are in hospital for other diseases and treatments, family members, and the community (10).

Standard, contact and airborne precautions

In a study of 1099 patients with confirmed COVID-19, only $1.9 \%$ had any contact with animals, while the majority of $72.3 \%$ had contact with residents of Wuhan indicating a high degree of community transmission. The most common symptoms were fever and cough developing within an average incubation period. Also, an overwhelming 83.2\% had lymphocytopenia indicating that the virus seems to directly affect the immune status (11).

Conventional routes of transmission of SARS-CoV-2 are thought to be similar to other contemporary coronavirus syndromes including SARS and MERS, including respiratory droplets and direct contact. However, detection of SARS-CoV-2 in the gastrointestinal tract, saliva, and urine may suggest other potential portals of transmission as well (11).

Transmission of COVID-19 is mainly through respiratory droplets from cough or sneeze. Droplets can be deposited in the mouths, noses, or eyes of people nearby or be inhaled into the lungs of those in close proximity. The possibility or degree of aerosol transmission is currently uncertain (3).

\section{Standard and contact precautions}

Blood, body fluids, secretions, excretions except sweat may contain transmissible infectious agents via nonintact skin and mucous membranes. Standard precautions are determined by the nature of the patient interaction and the extent of anticipated blood, body fluids, and pathogen exposure. If splashes, sprays, coughs, contact with nonintact skin, mucous membranes are expected, facemask, gloves, and eye protection should be worn (3).

Hand hygiene should be performed with alcohol-based hand rub containing at least $60 \%-95 \%$ alcohol or by washing hands with soap and water for at least 20 seconds. It should be done before and after all patient interaction or contact with potentially infectious material, and before putting on and after removing PPE.

All personnel should receive training on when and what PPE to use, how to properly wear and remove PPE in a manner to prevent self-contamination and how to dispose of and maintain PPE.

Respirator or facemask should be donned before entry into the patient area. Disposable respirators and facemasks should be discarded after exiting the patient's care area and closing the door. Hand hygiene should be performed after discarding the respirator or facemask.

Eye protection should be worn prior to entering the care area and removed before leaving the patient room. Reusable respirators and eye protection must be disinfected according to manufacturer's instructions.

Gloves are to be worn before entry to the patient area and are to be discarded when leaving, followed by immediate hand hygiene.

Gowns are mandated to be donned before entry into the procedure room and discarded in a dedicated container before leaving the procedure suite. Cloth gowns should be laundered after each use. In case of shortages, they should be prioritized for aerosol-generating procedures, procedures where splashes and sprays are anticipated, and high-contact patient care activities.

\section{Airborne precautions}

Procedures likely to induce coughing should be performed cautiously or avoided, if possible. If they have to be performed, adherence to the following guidelines can be helpful in limiting the spread. 
Procedures for patients under airborne precautions should be performed in an AlIR. In case of nonavailability of a negative-pressure room, a local exhaust ventilation device should be used. Only those personnel essential to the procedure and patient care should be present. All HCP in the room should wear an N95 or higher-level respirator, eye protection, gloves, and a gown. Prompt cleaning and disinfection should be undertaken (3).

The use of a particulate respirator requires both a fit test and regular user seal checks. If the user is unable to achieve a tight air seal with a particulate respirator or if the user has facial hair or certain medical conditions, PAPR must be worn when airborne precautions are in effect, the use of which requires additional training (9).

\section{Segregation of workforce}

Many departments have staff who provide clinical services at multiple locations. These cross-covering staff who has been in contact with a COVID-19 patient risk exposing multiple healthcare teams to the contagion. It is prudent to create a separate clinical team which includes the treating physician and an entire unit of nurse, technician, as well as other support staff, who are necessary for complete patient care at one particular center. Optimally, at least two such separate units should be created for one site so that care can be provided without interruption in case a member of the alternating team needs to go into isolation. It is also recommended that these units refrain from contact with each other and treat different subsets of patients (2).

\section{Temperature checking and condition of staff}

Many reports have shown that people of certain demographic parameters and those with comorbidities are at higher risk of acute respiratory distress syndrome (ARDS) if infected. Exclusion of IR staff with these parameters (age above 60, smokers, diabetics, hypertensives, those with previous lung damage, chronic heart disease, cancer, Hepatitis B, chronic kidney disease) from com- ing into contact with confirmed COVID-19 cases is prudent.

Although fever, dry cough and breathing difficulty, myalgia and fatigue are the most common symptoms, there have been documented instances of isolated symptoms of sore throat, sneezing and nasal discharge with a far less occurrence of headache, sputum production, hemoptysis, and diarrhea (10).

To prevent further spread, onset of fever and respiratory symptoms should be closely monitored among HCP. When available, testing of respiratory specimens should be done immediately once a diagnosis is suspected. Serum antibodies, fecal and urine samples should also be tested among HCP before and after their exposure to SARSCoV-2 for identification of asymptomatic infections (10).

\section{Quarantine and treatment of staff}

Symptomatic or RT-PCR positive members of the staff should be quarantined for 14 days. Exposed but asymptomatic HCP may continue to work after consultation with the occupational health program in case of limited essential workforce.

As a gold standard, respiratory specimens are tested for SARS-CoV-2 using RTPCR assays. Conservative management is the mainstay of treatment in uncomplicated cases with debatable benefits of corticosteroid therapy and antiviral drugs. The common complications identified include ARDS (29\%), RNAaemia (15\%), acute cardiac injury (12\%), and secondary infection (10\%) (10).

\section{Conclusion}

The IR department should be prepared to continue the service especially for emergency procedures and important elective procedures, during the time of increasing incidence of COVID-19 cases. The team members should understand the routes of transmission and should take utmost precautions to prevent transmission to colleagues and patients.

Anticipating that constant updates will be necessary in this volatile situation, the above recommendations may be used as a framework on which to base management decisions and strategize in order to optimize resources while reducing risk to staff and delivering quality patient care.

\section{Conflict of interest disclosure \\ The authors declared no conflicts of interest.}

\section{References}

1. Wu Z, McGoogan JM. Characteristics of and important lessons from the coronavirus disease 2019 (COVID-19) outbreak in China: Summary of a report of 72314 cases from the Chinese Center for Disease Control and Prevention. JAMA 2020 Feb 24. [Crossref]

2. Stempniak M. 6 steps interventional radiology can take to prepare for the coronavirus. Radiology Business February 27, 2020 [Online]. Available at https://www.radiologybusiness. com/topics/leadership/interventional-radiology-prepare-coronavirus Accessed: Mar 19, 2020.

3. CDC, Centers for Disease Control and Prevention. Coronavirus Disease 2019 (COVID-19), 07 Mar 2020. [Online]. Available at https://www. cdc.gov/coronavirus/2019-ncov/hcp/guidance-risk-assesment-hcp.html Accessed: Mar 19, 2020.

4. Wang $\mathrm{CJ}, \mathrm{Ng} \mathrm{CY}$, Brook RH. Response to COVID-19 in Taiwan: big data analytics, new technology, and proactive testing. JAMA 2020 Mar 3. [Crossref]

5. Zhu N, Zhang D, Wang W, et al. A novel coronavirus from patients with pneumonia in China, 2019. N Engl J Med 2020; 382:727-733. [Crossref]

6. Reddy P, Liebovitz D, Chrisman H, Nemcek Jr AA, Noskin GA. Infection control practices among interventional radiologists: results of an online survey. J Vasc Interv Radiol 2009; 20:1070-1074. [Crossref]

7. Abi-Jaoudeh N, Walser EM, Bartal G, et al. Ebola and other highly contagious diseases: strategies by the society of interventional radiology for interventional radiology. J Vasc Interv Radiol 2016; 27:200-202. [Crossref]

8. Malavaud S, Joffre F, Auriol J, Darres S. Hygiene recommendations for interventional radiology. Diagn Interv Imaging 2012; 93:813-822. [Crossref]

9. Mirza SK, Tragon TR, Fukui MB, Hartman MS, Hartman AL. Microbiology for radiologists: how to minimize infection transmission in the radiology department. Radiographics 2015; 35:1231-1244. [Crossref]

10. Huang C, Wang Y, Li X, et al. Clinical features of patients infected with 2019 novel coronavirus in Wuhan, China. Lancet 2020; 395:497-506. [Crossref]

11. Guan WJ, Ni ZY, Hu Y, et al. Clinical characteristics of coronavirus disease 2019 in China. N Engl J Med 2020 Feb 28. 